(C) 1996 IEEE. Personal use of this material is permitted. However, permission to reprint/republish this material

for advertising or promotional purposes or for creating new collective works for resale or redistribution to servers

or lists, or to reuse any copyrighted component of this work in other works must be obtained from the IEEE.

\title{
Study of Field Ionization in the Charge Exchange Injection for the IPNS Upgrade*
}

\author{
Yong-Chul Chae and Yanglai Cho \\ Argonne National Laboratory, 9700 So. Cass Ave., Argonne, Illinois 60439, U.S.A.
}

\section{Abstract}

The proposed 1-MW neutron spallation source is a rapidcycling synchrotron (RCS) with a design intensity of $1.04 \times 10^{14}$ protons. A $H^{-}$beam from the linac is injected into the synchrotron via the charge exchange process. Due to the high intensity of the beam, the minimization of beam loss is one of the primary concerns. In this paper, we study a possible beam loss associated with @eld ionization, which includes estimates of the charge fraction and level distribution of the excited hydrogen atoms after stripping, and an estimate of lifetime of the excited hydrogen atoms in the transverse magnetic ${ }^{\circledR}$ eld.

\section{INTRODUCTION}

Minimization of beam losses is one of the major goals at the proposed neutral spallation source at Argonne. Among the various injection-loss mechanisms, the beam loss due to ®eld ionization has recently received a great deal of attention after Hutson and Macek at Los Alamos reported that the measured 0.2-0.3\% of beam loss at the Proton Storage Ring (PSR) [1] was attributed to ®eld ionization of the $n \geq 3$ excited hydrogen atoms in the 1.2-Tesla bending magnet located downstream of the stripping foil.

If the foil strips the electrons of injected $\mathrm{H}^{-}$ions completely, we will not have the ®eld-ionization loss. However, for a given foil thickness, the stripping ef $\AA$ ciency is less than $100 \%$ resulting in the fraction of neutral hydrogen in the various excited states. It is these hydrogen atoms that will be ionized in the magnetic ®eld and, following a wrong orbit, eventually lost.

\section{FIEIIONIZA TION}

Let's consider an energetic hydrogen atom moving through the uniform magnetic ${ }^{\circledR}$ eld $\mathbf{B}$ whose direction is normal to the velocity $\mathbf{v}$. Magnetic ${ }^{\circledR}$ eld in the lab frame is transformed to mostly electric ®eld in the rest frame according to:

$$
F(V / m)=\gamma \beta c B(T),
$$

where $\gamma$ and $\beta$ are the usual relativistic quantities, $\mathrm{c}$ is the speed of light, $\mathrm{B}$ is the magnetic $®$ eld in the lab frame, and $\mathrm{F}$ is the electric ${ }^{\circledR} e l d$ in the rest frame. This external electric ${ }^{\circledR}$ eld puts the hydrogen atom in Stark states.

The Hamiltonian for the hydrogen atom in a Stark state may be written as

$$
\begin{aligned}
H & =H_{0}+H^{\prime} \\
H_{0} & =\frac{p^{2}}{2 m}-\frac{e^{2}}{r} \\
H^{\prime} & =-e F z=-e F r \cos \theta,
\end{aligned}
$$

Work supported by U.S. Department of Energy, Of®ce of Basic Energy Sciences under Contract No. W-31-109-ENG-38.

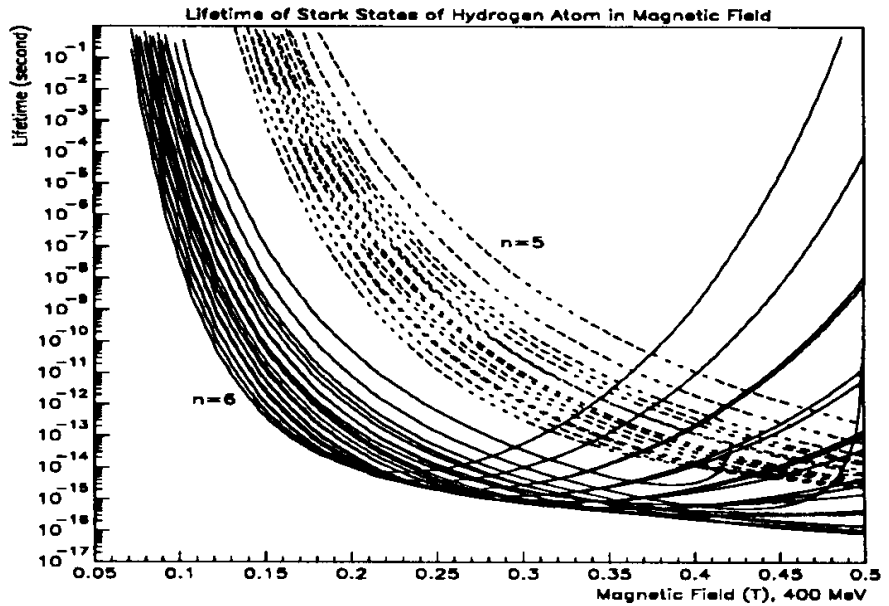

Figure 1

Lifetime of Stark States $(n=5,6)$ of Hydrogen Atom in the Magnetic Field at $400 \mathrm{MeV}$. (The group of lines represents $\mathrm{n}(\mathrm{n}+1) / 2$ energy states for a given $\mathrm{n}$.)

where we assume the external @eld is in the z-direction. Due to the external ®eld, the potential well of unperturbed hydrogen atoms is distorted in such a way that the width of the barrier becomes ${ }^{\circledR}$ nite, which in turn allows the possibility of ionization via tunneling.

In order to include the effect of ionization in the solution of the Schrìdinger equation, Landau [2] introduced the complex energy values de®ned as

$$
E=E_{0}-\frac{1}{2} i \Gamma
$$

where $E_{0}$ and $\Gamma$ are two constants, which are positive. The physical signi ${ }^{\circledR}$ cance of the complex energy can be seen by writing the time factor in the wave function of the form

$$
e^{-(i / \hbar) E t}=e^{-(i / \hbar) E_{0} t} e^{-(\Gamma / \hbar) t / 2} .
$$

It can be seen that the probability of @nding the electron inside the barrier decreases with time as $e^{-(\Gamma / \hbar) t}$. Thus $\Gamma$ determines the lifetime of the state de ${ }^{\circledR}$ ned by $\tau=\hbar / \Gamma$, whose relation satis ${ }^{\circledR}$ es Heisenberg's uncertainty relation. If we measure the energy state, the spectrum will be centered at $E_{0}$ with width $\Gamma$. In the literature $E_{0}$ is commonly called Stark energy and $\Gamma$ is linewidth (of the spectrum).

Damburg and Kolosov [3] solved Schrøidinger equation in the parabolic coordinate system ${ }^{1}$ for $E_{0}$ and $\Gamma$ using the perturbation method. They obtained the series in $F$ for the Stark energy and derived a semiempirical formula for $\Gamma$.

Damburg and Kolosov's formula was used to calculate the Stark energy and the lifetime of hydrogen atoms in excited states

\footnotetext{
${ }^{1}$ The choice of parabolic coordinates for the Stark-effect problem is not incidental. For a clear exposition of choosing a proper coordinate system, see $\mathrm{p}$. 1676 in Ref. [4]
} 
[5]. The results for $n$, the principal quantum number, equal to 5 and 6 are shown in Fig. 1, where the lifetimes of $n(n+1) / 2$ energy states for a given $n$ are plotted as a function of magnetic ßeld.

In the calculation, we assumed the injection energy of 400 $\mathrm{MeV}$ and considered the magnetic ßeld strength up to $0.5 \mathrm{~T}$. However, the ionization lifetime greater than $10^{-8} \mathrm{sec}$ should be interpreted carefully, for the average radiation transition lifetime of a hydrogen atom is of the order of $10^{-8} \mathrm{sec}$ or greater for $n \geq 3[6]$

\section{Charge Fraction of $H^{-}$IONS}

When $H^{-}$ions traverse a carbon foil, charge exchange processes occur. Assuming that the electron capturing process, governed by the $E^{-3}$ law, is negligible, there are three important electron loss processes:

(i) $H^{-} \rightarrow H^{0}$, with cross section $\sigma_{-10}$,

(ii) $H^{-} \rightarrow H^{+}$, with cross section $\sigma_{-11}$,

(iii) $H^{0} \rightarrow H^{+}$, with cross section $\sigma_{01}$.

In terms of these cross sections, the charge fractions can be written as

$$
\begin{aligned}
N_{H^{-}} & =e^{-\left(\sigma_{-10}+\sigma_{-11}\right) x}, \\
N_{H^{0}} & =\frac{\sigma_{-10}}{\left(\sigma_{-10}+\sigma_{-11}\right)-\sigma_{01}}\left[e^{-\sigma_{01} x}-e^{-\left(\sigma_{01}+\sigma_{-11}\right) x}\right], \\
N_{H^{+}} & =1-N_{H^{-}}-N_{H^{0}},
\end{aligned}
$$

where $x$ is the foil thickness (the number of target atoms $/ \mathrm{cm}^{2}$ ), and $N_{H^{-}}, N_{H^{0}}$ and $N_{H^{+}}$are the three charge fractions in the beam.

Theoretical calculations for electron loss cross section have been worked out by several authors. One of these theories is due to Gillespie [7]. His results agreed well with the measurements in the wide range of energy including the measurements done at Fermilab with $200-\mathrm{MeV}$ beam and the one at Los Alamos with $800-\mathrm{MeV}$ beam. These measured cross sections are presented in Table 1 , which indicates that cross section varies as $\beta^{-2}$. Gillespie's theory also shows such a scaling law ${ }^{2}$.

Table 1

Electron Loss Cross Sections.

\begin{tabular}{|l|c|c|}
\hline $\begin{array}{l}\text { Kinetic Energy } \\
(\mathrm{MeV})\end{array}$ & $\begin{array}{c}\sigma_{-10}+\sigma_{-11} \\
\left(\times 10^{-18} \mathrm{~cm}^{2}\right)\end{array}$ & $\begin{array}{c}\sigma_{01} \\
\left(\times 10^{-18} \mathrm{~cm}^{2}\right)\end{array}$ \\
\hline 200 (Measured, [8]) & $1.56 \pm 0.14$ & $0.60 \pm 0.10$ \\
\hline 400 (Fitted) & 0.98 & 0.38 \\
\hline 800 (Measured, [9]) & 0.67 & 0.33 \\
\hline
\end{tabular}

But both sets of data with beam at $200 \mathrm{MeV}$ and $800 \mathrm{MeV}$ show slightly smaller values than the theory [8]. In order to estimate the cross section for $400-\mathrm{MeV}$ beam, instead of using the theoretical result directly (which may be all right), we ${ }^{\circledR} t$ two measurement data with the $\beta^{-2}$ scaling law. The result obtained

\footnotetext{
${ }^{2}$ Stopping power of the foil is also governed by the same scaling law, which indicates that the two processes are similar. In fact, both processes are dominated by electron-electron elastic scattering.
}

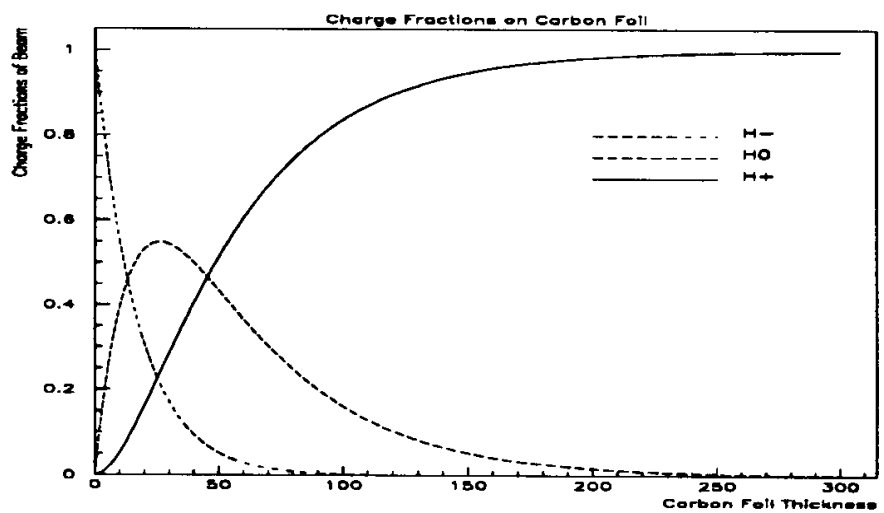

Figure 2

Charge Fractions vs. Foil Thickness at $400 \mathrm{MeV}$.

is also included in Table 1. The accuracy of this ${ }^{\circledR} t$ is within one standard deviation of measurement.

Substituting the estimated cross section into Eq. (5), we obtain the charge fractions as functions of foil thickness. These results are shown in Fig. 2. Numerical values for the interesting range of foil thicknesses are also summarized in Table 2.

Table 2

Charge Fraction after Carbon Foil of Various Thicknesses.

\begin{tabular}{|c|c|c|c|}
\hline $\begin{array}{c}\text { Foil Thickness } \\
\left(\mu \mathrm{g} / \mathrm{cm}^{2}\right)\end{array}$ & $\begin{array}{c}N_{H^{-}} \\
(\%)\end{array}$ & $\begin{array}{c}N_{H^{0}} \\
(\%)\end{array}$ & $\begin{array}{c}N_{H^{+}} \\
(\%)\end{array}$ \\
\hline 200 & 0.0007 & 1.7 & 98.3 \\
\hline 210 & 0.0004 & 1.3 & 98.7 \\
\hline 220 & 0.0002 & 1.1 & 98.9 \\
\hline 230 & 0.0001 & 0.85 & 99.15 \\
\hline 240 & 0.00007 & 0.67 & 99.33 \\
\hline 250 & 0.00004 & 0.54 & 99.46 \\
\hline
\end{tabular}

\section{IV. $n$-DISTRIBUTION}

After the foil, the neutral hydrogen atoms are distributed (or populated) in different states. At present no theory exists for excited-state production using the beam-foil method. However, we may mention the atomic-collision theory for radiative capture of free electrons by bare nuclei in the high velocity limit, which shows $n^{-3}$ dependence on the principal quantum number of the capture cross section [6]. Even if it is not clear whether we can apply this approach to beam-foil interaction, the early measurement results at low energy (less than $1 \mathrm{MeV} / \mathrm{au}$ ) showed such a dependence on principal quantum number ${ }^{3}$. An interesting theoretical analysis [11] of post-foil measurement of electromagnetic radiation and ion charge also suggests that the level populations decrease as $n^{-3}$ and depend universally on the kinetic energy of the incoming beam. From these early studies we learn [11]:

-dependence of the level population on principal quantum number according to $n^{-3}$ is observed frequently but not exclu-

\footnotetext{
${ }^{3}$ June Davidson [10] used neutral helium at $0.275 \mathrm{MeV}$ in order to measure the absolute population in $3 \leq n \leq 6$ after $6 \mu \mathrm{g} / \mathrm{cm}^{2}$ carbon foil.
} 
sively,

-dependence of the level population on foil thickness is unknown,

-dependence of the level population on kinetic energy of the incident beam was not observed.

Since the above studies are not conclusive enough to apply the ${ }^{\circledR}$ ndings to $400 \mathrm{MeVH}^{-}$ions passing the carbon foil as proposed for the neutral spallation source at Argonne, we pay attention to the recent experimental study on beam-foil interaction [12]. Assuming that the $\mathrm{n}$ distribution is governed by a power law $n^{-p}$, the exponent of the power law $p$ is measured for a given foil at $800 \mathrm{MeV}$. The results are found to be:

for a given $25.0 \mu \mathrm{g} / \mathrm{cm}^{2}$ carbon foil

$$
\begin{cases}p=3.41 & \text { for } \mathrm{n}=2, \ldots, 5 \\ p=8.0 & \text { for } \mathrm{n}=10, \ldots, 14\end{cases}
$$

for a given $198.0 \mu \mathrm{g} / \mathrm{cm}^{2}$ carbon foil

$$
\begin{cases}p=1.29 & \text { for } \mathrm{n}=2, \ldots, 5 \\ p=8.0 & \text { for } \mathrm{n}=10, \ldots, 14 .\end{cases}
$$

It is interesting to note that a single power law is unable to characterize the $n$ distribution of excited states over a wide range of $n$ and the low-lying states become more evenly populated for the thicker foils.

\section{M.PPLICA TION}

The injection orbit in the IPNS-Upgrade RCS [13] is shown in Fig. 3. With a $250-\mu \mathrm{g} / \mathrm{cm}^{2}$ stripper foil, about $0.54 \%$ of the $H^{-}$beam emerges from the foil as partially stripped neutral hydrogen atoms, some of which are in the ground state and some of which are in excited states. If these particles are allowed to enter a normal bending magnet @eld, they will become stripped and either hit the vacuum chamber wall or, if not lost, form a halo of large betatron oscillation around the normal proton beam.

Figure 3 shows that the neutrals pass through the center of one quadrupole (QD) and enter the next quadrupole $(\mathrm{QF})$ at $-11 \mathrm{~cm}$, where the ®eld is $0.3 \mathrm{~T}$, unless the $H^{0}$-catcher is installed. This ®eld over a length of $0.5 \mathrm{~m}$ is enough to strip all electrons with $n \geq 5$, or about $20 \%$ of the $H^{0}$ beam. In this estimation, we assumed that $n$-distribution follows $n^{-p}$ dependence, and, since the foil thickness is $250 \mu \mathrm{g} / \mathrm{cm}^{2}$, we used $p=1.29$ for the conservative estimate. The catcher, therefore, is placed upstream of this quadrupole (QF) as shown in Fig. 3.

The relatively short bumper magnets, B3 and B4 shown in Fig. 3, can also ionize the $H^{0}$ beam. However, the beam loss due to the ${ }^{\circledR}$ eld ionization in these bumper magnets is negligible.

\section{VC.ONCLUSION}

In order to minimize the beam loss in the proposed IPNSUpgrade RCS due to ®eld ionization, we propose to use the relatively thick stripper foil with thickness of $250 \mu \mathrm{g} / \mathrm{cm}^{2}$ and to install a $H^{0}$-catcher in the ring together with the careful trajectory control of the neutral hydrogen atoms.

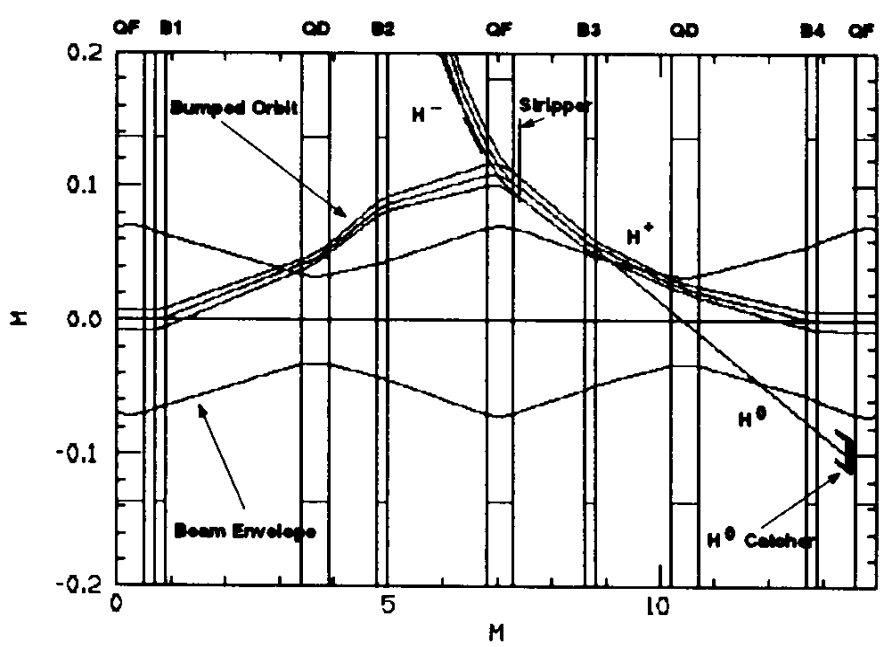

Figure 3

Injection Orbit in the IPNS-Upgrade RCS.

\section{VIR.EFERENCES}

[1] R. Hutson and R. Macek, Proc. 1993 Part. Accel. Conf., 363, 1993.

[2] L. D. Landau and E. M. Lifshitz, Quantum Mechanics 3rd Ed., Pergamon Press, 1977.

[3] R. J. Damburg and V. V. Kolosov, in Rydberg States of Atoms and Molecules, edited by R. F. Stebbings, Cambridge University Press, 1983.

[4] P. M. Morse and H. Feshbach, Methods of Theoretical Physics, McGraw-Hill Book Company, 1953.

[5] Y.-C. Chae, ANL Report NSA-94-2, 1994.

[6] H. A. Bethe and E. E. Salpeter, Quantum Mechanics of One- and Two-Electron Atoms, Plenum Pub. Corp., 1977.

[7] G. H. Gillespie, Phys. Rev., A 16, 943, 1977.

[8] R. C. Webber and C. Hojvat, IEEE Trans. Nucl. Sci., NS26, No. 3, 4012, 1979.

[9] O. B. van Dick et al., AIP Conf. Proc. No. 69, 985, 1980.

[10] June Davidson, Phys. Rev., A 12, 1350, 1975.

[11] T. Aberg and O. Goscinki, Phys. Rev., A 24, 801, 1982, and references therein.

[12] A. H. Mohagheghi et al., Phys. Rev., A 43, 1345,1991.

[13] IPNS Upgrade-A Feasibility Study, ANL-95/13 (Draft), April 1995. 